\title{
Kink propagation and trapping in a two-dimensional curved Josephson junction
}

Gorria, Carlos; Gaididei, Yuri Borisovich; Sørensen, Mads Peter; Christiansen, Peter Leth; Caputo, Jean Guy

Published in:

Physical Review B Condensed Matter

Link to article, DOI:

10.1103/PhysRevB.69.134506

Publication date:

2004

Document Version

Publisher's PDF, also known as Version of record

Link back to DTU Orbit

Citation (APA):

Gorria, C., Gaididei, Y. B., Sørensen, M. P., Christiansen, P. L., \& Caputo, J. G. (2004). Kink propagation and trapping in a two-dimensional curved Josephson junction. Physical Review B Condensed Matter, 69(13), 134506. https://doi.org/10.1103/PhysRevB.69.134506

\section{General rights}

Copyright and moral rights for the publications made accessible in the public portal are retained by the authors and/or other copyright owners and it is a condition of accessing publications that users recognise and abide by the legal requirements associated with these rights.

- Users may download and print one copy of any publication from the public portal for the purpose of private study or research.

- You may not further distribute the material or use it for any profit-making activity or commercial gain

- You may freely distribute the URL identifying the publication in the public portal 


\title{
Kink propagation and trapping in a two-dimensional curved Josephson junction
}

\author{
C. Gorria, ${ }^{1,2}$ Yu. B. Gaididei, ${ }^{1,3}$ M. P. Soerensen,,${ }^{1}$ P. L. Christiansen, ${ }^{1}$ and J. G. Caputo ${ }^{4}$ \\ ${ }^{1}$ Section of Mathematical Physics, IMM, Technical University of Denmark, DK-2800, Kongens Lyngby, Denmark \\ ${ }^{2}$ Department of Applied Mathematics and Statistics, University of the Basque Country, E-48080 Bilbao, Spain \\ ${ }^{3}$ Bogolyubov Institute for Theoretical Physics, 252143 Kiev, Ukraine \\ ${ }^{4}$ Laboratoire de Mathématiques, INSA de Rouen, Boîte Postale 08, 76131 Mont-Saint-Aignan Cedex, France \\ and Laboratoire de Physique Théorique et Modelisation, Université de Cergy-Pontoise and CNRS, France
}

(Received 8 October 2003; published 16 April 2004)

\begin{abstract}
Sine-Gordon kink propagation in a curved planar waveguide is considered. The waveguide consists of two rectangular regions joined by a bent section of constant curvature. Transverse homogeneous and inhomogeneous Neumann boundary conditions are used. The latter models an energy-providing mechanism for Josephson junctions of overlap type. A collective variable approach based on the kink position and the kink width depending on the transversal coordinate is developed. The latter allows to take into account both longitudinal and centrifugal forces which act on the nonlinear excitation moving in a region with finite curvature and to obtain a qualitatively good agreement with the numerical simulations. The region with finite curvature acts as a potential barrier whose height and width depend on the radius of curvature of the waveguide. The kink transmission, reflection, and trapping are investigated. The kink may be captured when a driving force, provided by a magnetic field, is applied to the kink.
\end{abstract}

DOI: 10.1103/PhysRevB.69.134506

PACS number(s): 74.50. $+\mathrm{r}, 05.45 . \mathrm{Yv}, 85.25 . \mathrm{Cp}$

\section{INTRODUCTION}

Recent advances in microstructuring technology have made it possible to fabricate various low-dimensional systems with complicated geometry. Examples are photonic crystals with embedded defect structures such as microcavities, waveguides, and waveguide bends $;{ }^{1}$ narrow constructions (quantum dots and channels) formed at semiconductor heterostructures, ${ }^{2}$ magnetic nanodisks, dots, and rings, ${ }^{3,4}$ etc.

It is well known that the wave equation subject to Dirichlet boundary conditions has bound states in straight channels of variable width ${ }^{5}$ and in curved channels of constant cross section. ${ }^{6}$ Spectral and transport characteristics of quantum electron channels ${ }^{7}$ and waveguides in photonic crystal ${ }^{8}$ are essentially modified by the existence of segments with finite curvature.

Until recently there have been a few theoretical and numerical studies of the effect of curvature on properties of nonlinear excitations. Nonlinear whispering gallery modes for a nonlinear Maxwell equation in a microdisk were investigated in Ref. 9, the excitation of whispering-gallery-type electromagnetic modes by a moving fluxon in an annular Josephson junction was found in Ref. 10. The fluxon dynamics in exponentially tapered Josephson flux-flow oscillator was studied in Ref. 11. Nonlinear localized modes in twodimensional photonic crystal waveguides were investigated in Ref. 12. A curved chain of nonlinear oscillators was considered in Ref. 13 and it was shown that the interplay of curvature and nonlinearity leads to a symmetry breaking when an asymmetric stationary state becomes energetically more favorable than a symmetric stationary state. Propagation of Bose-Einstein condensates in magnetic waveguides was found quite recently in Ref. 14. Single-mode propagation was observed along homogeneous segments of the waveguide while geometric deformations of the microfabricated wires lead to strong transverse excitations.
The aim of this paper is to study the motion of fluxons moving in a two-dimensional finite domain. Specifically we treat a planar curved Josephson junction whose width is constant and is much smaller than its entire length. We consider inhomogeneous Neumann boundary conditions on the transverse boundaries of the domain. Using a simple collective variable analysis based on the fluxon position we show that a region of nonzero curvature in a waveguide induces a potential barrier for the wave. This is different from the case of transverse Dirichlet boundary conditions where studies on the (linear) Schrödinger equation show the existence of a localized mode which will trap waves in the curved region. The paper is organized as follows. Section II describes the model. In Sec. III a collective coordinate approach is developed. The results of numerical simulations and their comparison with the results of the collective coordinate approach are discussed in Sec. IV. Finally, Sec. V contains our conclusions.

\section{MODEL AND EQUATIONS OF MOTION}

We consider a two-dimensional Josephson junction $\Omega$ constructed of two straight segments joined by a bent section (see Fig. 1). The fluxon dynamics can be described by the two-dimensional sine-Gordon (SG) equation

$$
\frac{\partial^{2} \phi}{\partial t^{2}}-\frac{\partial^{2} \phi}{\partial x^{2}}-\frac{\partial^{2} \phi}{\partial y^{2}}+\alpha \frac{\partial \phi}{\partial t}+\sin \phi=0
$$

where $\alpha$ is the damping coefficient. The boundary conditions for Eq. (1) are obtained from the relation ${ }^{15,16}$ between the effective magnetic field $\mathcal{H}$ and the phase difference $\phi$ by means of the relation

$$
\overrightarrow{\mathcal{H}}=-\hat{z} \times \nabla \phi,
$$




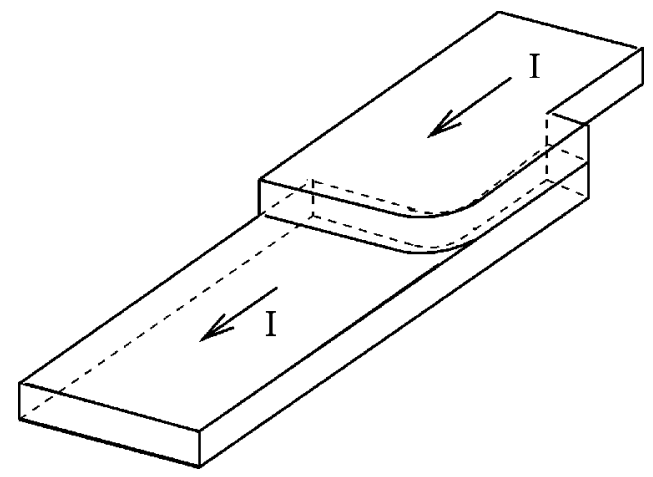

FIG. 1. Curved Josephson junction structure.

where $\hat{z}$ is the unit vector normal to the junctions plane, and have the form

$$
\left.\vec{n} \cdot \boldsymbol{\nabla} \phi\right|_{\partial \Omega}=\left.\vec{n} \cdot(\hat{z} \times \overrightarrow{\mathcal{H}})\right|_{\partial \Omega} .
$$

Here $\vec{n}$ is the outward normal to the boundary of the junction region $\partial \Omega$.

In this paper $\Omega$ has constant width $w$ (see Fig. 2). ${ }^{17}$ The center curve in the bent and straight sections of $\Omega$, denoted $\mathcal{C}$, is given by $\vec{r}=\vec{\rho}(s) \equiv[a(s), b(s)]$, where $s$ is the arc length of $\mathcal{C}$. Note the normalization $\left\|\vec{\rho}^{\prime}(s)\right\|=1$, where prime denotes derivative with respect to $s$. The points in $\Omega$ are labeled in accordance with the parametrization

$$
\vec{r}=\vec{\rho}(s)+u \vec{n}(s)
$$

where the coordinate $u$ measures the signed distance from $\mathcal{C}$. $\vec{n}(s)=\left[-b^{\prime}(s), a^{\prime}(s)\right]$ denotes the unit normal to $\mathcal{C}$. To satisfy the normalization assumption we choose $a^{\prime}(s)$ $=\cos [\theta(s)], b^{\prime}(s)=\sin [\theta(s)]$. The parametrization (4) implies that $(s, u)$ are orthogonal coordinates and the components of the metric tensor are

$$
g_{s s}=[1-u \kappa(s)]^{2}, \quad g_{u u}=1,
$$

where $\kappa(s)=\sqrt{\left(a^{\prime \prime}\right)^{2}+\left(b^{\prime \prime}\right)^{2}}$ is the curvature of the reference line. In this case the curvature of the reference curve $\mathcal{C}$ takes the form $\kappa(s)=\theta^{\prime}(s)$. The curve $\mathcal{C}_{-}\left(\mathcal{C}_{+}\right)$which is given by Eq. (4) with $u=-w / 2(u=w / 2)$ represents the inner boundary (outer boundary) of the junction.

In the case when the external magnetic field is caused by a current passing through the junction, then inhomogeneous Neumann boundary conditions are induced for the overlap geometry and they may be written in the form of

$$
\left.\frac{\partial \phi}{\partial u}\right|_{u= \pm w / 2}= \pm \frac{\mathcal{I}}{2 \ell_{ \pm}}
$$

where $\mathcal{I}$ is the normalized total bias through the junction and $\ell_{ \pm}$is the normalized length of the boundary $\mathcal{C}_{ \pm}$. Assuming that $\ell_{ \pm} \gg w$, we will neglect the difference between the length of the inner and outer boundaries and replace $\ell_{ \pm}$by $\ell$ in Eq. (6).

In the curvilinear $(s, u)$ coordinates the $\mathrm{sG}$ equation (1) takes the form

$$
\frac{\partial^{2} \phi}{\partial t^{2}}-\frac{1}{g} \frac{\partial}{\partial s}\left(\frac{1}{g} \frac{\partial \phi}{\partial s}\right)-\frac{1}{g} \frac{\partial}{\partial u}\left(g \frac{\partial \phi}{\partial u}\right)+\alpha \frac{\partial \phi}{\partial t}+\sin (\phi)=0,
$$

where $g \equiv \sqrt{g_{s s} g_{u u}}=1-u \kappa(s)$ is the Jacobian of the transformation from the Cartesian coordinates to the $(s, u)$ coordinates, with the boundary conditions (6) and

$$
\partial_{s} \phi \rightarrow 0 \quad \text { for } \quad s \rightarrow \pm \infty .
$$

Let $\mathcal{C}$ be straight on both ends, $s<s_{1}$ and $s>s_{2}$, and let it have a finite constant curvature inside this interval

$$
\kappa(s)= \begin{cases}\frac{1}{R} & \text { when } \quad s_{1} \leqslant s \leqslant s_{2} \\ 0 & \text { elsewhere. }\end{cases}
$$

The corresponding expression for $\theta$ in the bent region is $\theta(s)=(\pi / 2)\left(s-s_{1}\right) /\left(s_{2}-s_{1}\right)$.

We will consider the scattering and trapping of kinks as they pass through the bending region.

\section{A COLLECTIVE COORDINATE APPROACH}

We will use the method of collective coordinates to gain understanding of how the bending affects the kink dynamics. It is not convenient to apply the collective coordinate approach in the case of inhomogeneous von Neumann boundary conditions. We use a trick similar to the one which was proposed in Ref. 18 for the case of rectangular Josephson junctions of overlap geometry. To this end we introduce an auxiliary function $\Phi_{0}(u)$ which satisfies the equation

$$
\frac{d^{2} \Phi_{0}}{d u^{2}}-\sin \Phi_{0}=0
$$

with the boundary condition

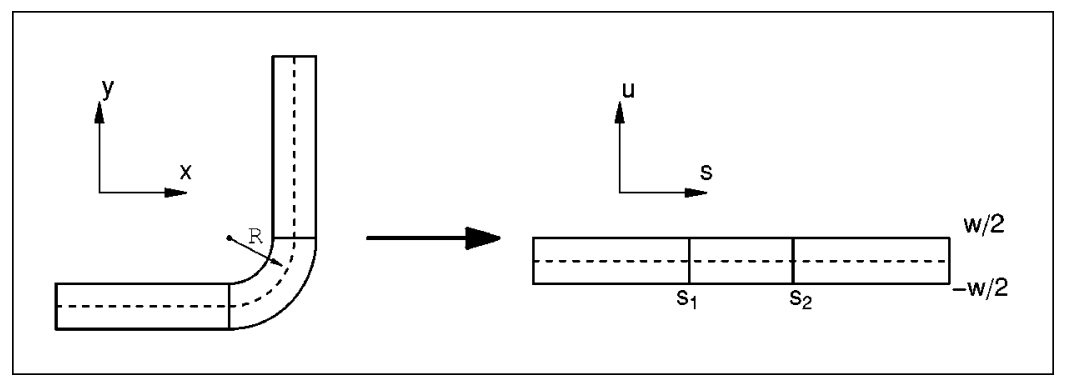

FIG. 2. Change from Cartesian to curvilinear coordinates. 


$$
\left.\frac{d \Phi_{0}}{d u}\right|_{u= \pm w / 2}= \pm \frac{\mathcal{I}}{2 \ell} .
$$

Now, a solution of the form

$$
\phi(s, u, t)=\Phi_{0}(u)+\psi(s, u, t)
$$

reduces Eqs. (6), (7), and (8) to

$$
\begin{gathered}
\frac{\partial^{2} \psi}{\partial t^{2}}-\frac{1}{g} \frac{\partial}{\partial s}\left(\frac{1}{g} \frac{\partial \psi}{\partial s}\right)-\frac{1}{g} \frac{\partial}{\partial u}\left(g \frac{\partial \psi}{\partial u}\right)+\alpha \frac{\partial \psi}{\partial t}+\sin \left(\psi+\Phi_{0}\right) \\
-\sin \Phi_{0}-\frac{1}{g} \frac{\partial g}{\partial u} \frac{d \Phi_{0}}{d u}=0,
\end{gathered}
$$

with the homogeneous von Neumann boundary conditions

$$
\left.\frac{\partial \psi}{\partial u}\right|_{u= \pm w / 2}=0
$$

for the transversal coordinate $u$ and the boundary conditions for the arclength coordinate $s$ identical to Eq. (8),

$$
\partial_{s} \psi \rightarrow 0 \text { for } s \rightarrow \pm \infty \text {. }
$$

In the absence of damping ( $\alpha=0$ ) the $\mathrm{sG}$ equation (13) may be obtained by variation of the Lagrange function

$$
L\{\phi\}=\int_{-\infty}^{\infty} \int_{-w / 2}^{w / 2} \frac{1}{w} \mathcal{L} d u d s
$$

where the Lagrangian density $\mathcal{L}$ can be represented as the following sum:

$$
\mathcal{L}=\mathcal{L}_{0}+\mathcal{L}_{d}
$$

The first term represents the density in the case without damping,

$$
\mathcal{L}_{0}=\left\{\frac{1}{2}\left(\frac{\partial \psi}{\partial t}\right)^{2}-\frac{1}{2 g^{2}}\left(\frac{\partial \psi}{\partial s}\right)^{2}-\frac{1}{2}\left(\frac{\partial \psi}{\partial u}\right)^{2}-[1-\cos (\psi)]\right\} g,
$$

while the second is

$$
\begin{aligned}
\mathcal{L}_{d}= & {\left[\left(1-\cos \Phi_{0}\right)(1-\cos \psi)-\sin \Phi_{0} \sin \psi\right.} \\
& \left.-s \frac{\partial \psi}{\partial s} \sin \Phi_{0}+\frac{1}{g} \frac{\partial g}{\partial u} \frac{d \Phi_{0}}{d u} \psi\right] g,
\end{aligned}
$$

and describes driving effects in the fluxon dynamics.

The starting point of the collective coordinate method is to choose a localized self-similar trial function which is close to the exact solution in the absence of bending and contains a number of parameters which become time dependent due to the perturbations. In our case, the trial function may be chosen as

$$
\psi(s, u, t)=4 \arctan \exp \left(\frac{s-\mathcal{S}(u, t)}{\mathcal{B}(u, t)}\right)
$$

where the functions $\mathcal{B}(u, t)$ and $\mathcal{S}(u, t)$ determine the width and the position, respectively, of the kink. From Eq. (14) we get the boundary conditions

$$
\left.\frac{\partial \mathcal{B}}{\partial u}\right|_{u= \pm w / 2}=\left.\frac{\partial \mathcal{S}}{\partial u}\right|_{u= \pm w / 2}=0
$$

To take explicitly into account the boundary conditions (21) we expand the position of the wave front $\mathcal{S}(u, t)$ and the width $\mathcal{B}(u, t)$ in Fourier series,

$$
\begin{aligned}
\mathcal{S}(u, t)= & \sum_{n=0}^{\infty}\left\{S_{2 n}(t) \cos \left[\frac{2 n \pi u}{w}\right]\right. \\
& \left.+S_{2 n+1}(t) \sin \left[\frac{(2 n+1) \pi u}{w}\right]\right\}, \\
\mathcal{B}(u, t)= & \sum_{n=0}^{\infty}\left\{B_{2 n}(t) \cos \left[\frac{2 n \pi u}{w}\right]\right. \\
& \left.+B_{2 n+1}(t) \sin \left[\frac{(2 n+1) \pi u}{w}\right]\right\} .
\end{aligned}
$$

Here the function $S_{0}(t)$ describes the motion of the flat front while the functions $S_{n}(t)(n \neq 0)$ characterize the curvature of the front. The functions $B_{n}(t)$ give the dependence of the width on time. Inserting Eqs. (20), (22), and (23) into Eqs. (16)-(19) and carrying out the integrations over $s$ and $u$ we obtain an effective Lagrangian in the form (see Appendix A for details)

$$
L=L_{0}+L_{d},
$$

being

$$
L_{0}=T-V
$$

where

$$
T=4\left(\dot{S}^{2}+\frac{\pi^{2}}{24} \dot{B}^{2}\right)
$$

is the effective kinetic energy, and

$$
\begin{aligned}
V= & 4\left\{\left(\frac{\pi^{4}}{24 w^{2}}+\frac{1}{2}\right) B^{2}+a_{0} U-\left[\left(\frac{2 w}{\pi^{2} R}-a_{1}\right) F\right.\right. \\
& \left.\left.+\left(\frac{2 w}{\pi^{2} R}+a_{1}\right) U\right] B\right\}
\end{aligned}
$$

is the effective potential energy. Finally

$$
L_{d}=-8 \gamma S \text {. }
$$

In Eqs. (24)-(28), $S(t) \equiv S_{0}(t), \quad B(t) \equiv B_{1}(t)$, and the following expressions have been used: 


$$
\begin{gathered}
U=\frac{\sinh (2 a)}{\cosh (2 a)+\cosh (2 S)}, \\
F=\frac{a+S}{2 \cosh ^{2}(S+a)}+\frac{a-S}{2 \cosh ^{2}(S-a)}, \\
a_{0}=-1+\frac{R}{w} \ln \frac{2 R+w}{2 R-w}, \\
\gamma=\left.\frac{\pi}{2 w} \frac{\partial \phi}{\partial u}\right|_{u=w / 2} \equiv \frac{x}{1-x} \sin \left(\frac{\pi R}{w} x\right) d x \\
a_{1}
\end{gathered}
$$

Here $2 a=s_{2}-s_{1}$ is the length of the bent region.

By deriving the Lagrange function (24)-(28) the following approximations were used.

(1) In the expansion (22) only the harmonics with $n=0$ are taken into account. This means that we neglected the front curvature [we checked the accuracy of this approximation and found that in the case when the width of the junction is not too large $(w \leqslant 2 \pi)$ the discarded terms do not contribute significantly to the kink dynamics].

(2) In the expansion (23) we substitute $\mathcal{B}(u, t)=1$ $+B(t) \sin (\pi u / w)$, which means that we neglected the zeroth harmonics and put $B_{0}(t)=1$ (remember that in the case of the straight waveguide $B_{0}=\sqrt{1-\dot{S}^{2}}$ ), and discarded the harmonics with $n \geqslant 1$. As expected we found this approximation to be correct for slow moving kinks $(\dot{S} \ll 1)$ and moderately wide waveguides $(w<2 \pi)$.

(3) Since we are interested in slow moving kinks $(\dot{S}$ $\ll 1$ ) we neglected the coupling terms of the type $\dot{S} \dot{B}$.

(4) Taking into account that we are interested here in the weak driving case $(\gamma<1)$ only the linear driving terms were considered. We also neglected the difference between the driving in the straight and bent part of the junction.

When the results of more sophisticated approach based on the four variational parameters $S_{0}, S_{1}, B_{0}$, and $B_{1}$ (see Appendix A) are compared with that of Eqs. (25)-(27), it is apparent that the simple approach catches all essential features of the kink dynamics.

\section{NUMERICAL AND ANALYTICAL RESULTS}

\section{A. Case without driving and damping}

From the Lagrangian (25)-(27) we obtain the equations of motion in the form

$$
\begin{aligned}
& 8 \ddot{S}+\frac{\partial V}{\partial S}=0, \\
& \frac{\pi^{2}}{3} \ddot{B}+\frac{\partial V}{\partial B}=0 .
\end{aligned}
$$

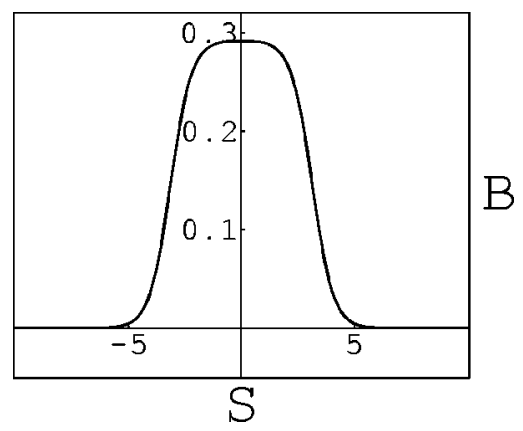

FIG. 3. Solution to Eq. (32) with $V$ given by Eq. (27) plotted in $(S, B)$ plane for $w=4, R=4, s_{2}=-s_{1}=\pi$.

Thus in the collective coordinate approach the kink dynamics in a curved waveguide is equivalent to the dynamics of a particle moving in the two-dimensional potential $V$. It is necessary to stress that considering the soliton width $\mathcal{B}(u, t)$ as a variational parameter in the trial function (20) is crucial. This variational parameter takes into account the centrifugal force which acts on the kink in the bending region. The width of the soliton in its stationary state $B_{s t}$ is given by the equation

$$
\frac{\partial V}{\partial B}=0
$$

and its value is

$$
B_{s t}=\frac{\left[2 w /\left(\pi^{2} R\right)-a_{1}\right] F+\left[2 w /\left(\pi^{2} R\right)+a_{1}\right] U}{1+\pi^{4} /\left(12 w^{2}\right)}
$$

This quantity is finite inside the bending region and rapidly vanishes outside of it (see Fig. 3). An illustration of how the presence of the second variational parameter affects the kink dynamics is given in Figs. 4 and 5. The latter represents the two-dimensional behavior of the potential function along the path $B=B_{s t}$. The figures show clearly that the potential profile which corresponds to the case of the one-parametric trial function $(B=0)$ represents a simple barrier. On the other hand, moving along the path $B=B_{s t}$ the particle experiences a potential profile with two humps separated by a valley between them. This potential well is shallow when $w \leqslant R$ and

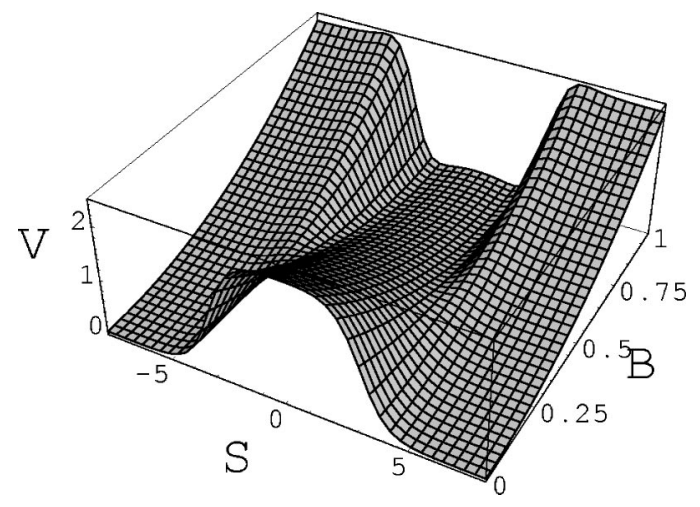

FIG. 4. The effective potential $V$ given by Eq. (27) for an equivalent mechanical system with $w=6, R=4, s_{2}=-s_{1}=\pi$. 


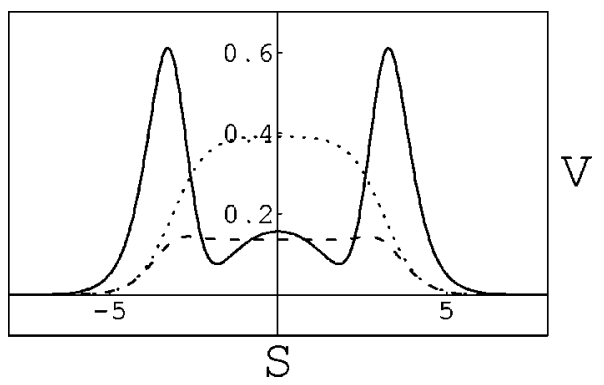

FIG. 5. Effective potential projection along the path $B=0$ for $w=4, R=4, s_{2}=-s_{1}=\pi$ (doted line) and along the path $B=B_{s t}$ for $w=6, R=4, s_{2}=-s_{1}=\pi$ (continuous line) and for $w=4, R=4, s_{2}$ $=-s_{1}=\pi$ (dashed line).

becomes deep and well pronounced when $w \rightarrow 2 R$. However, it is worth noting that the case $w \approx 2 R$ is beyond the accuracy of our approximations as the condition $|\mathcal{B}-1| \ll 1$ is violated when $w$ is close to $2 R$.

The numerical simulations of Eq. (1) have been conducted in the Cartesian coordinates $(x, y)$ using the finite element program package FEMLAB. ${ }^{19}$ On the triangular elements we have used Lagrange-linear and Lagrange-quadratic basis functions to expand the solution numerically. The expansion coefficients are time dependent and are governed by a system of ordinary differential equations (ODE's) derived from a variational formulation of Eq. (1). This system is automatically set up by FEMLAB and have been solved numerically employing the Matlab ordinary differential equation solvers ode15s and ode45. The ode15s is a multistep method solver of variable order designed for stiff systems and ode45 is an explicit Runge-Kutta method of order four and five. Specifying relative tolerances of $10^{-6}-10^{-3}$ gave satisfactory accurate numerical solutions with about 3800 elements in the domain at Fig. 2. We found that the nonstiff solver ode 45 is faster than ode $15 \mathrm{~s}$ and sufficiently efficient and accurate for the sine-Gordon equation (1). Typical run times are $9 \mathrm{~h}$ with ode 45 on a SUN Fire 3800, simulating from $t=0$ to $t=200$ with about 3800 elements and relative tolerance $10^{-3}$, absolute tolerance $10^{-4}$.

Initially the kink is located in the straight part of the domain far away from the domain edge and from the curved region to avoid interference with the boundaries. It is launched into the strip with different initial velocities. So as initial conditions for our numerical simulations we choose the function

$$
\phi(s, u, 0)=4 \arctan \exp \left(\frac{s-X_{0}}{\sqrt{1-v_{0}^{2}}}\right)
$$

and $\partial_{t} \phi(s, u, 0)=-v_{0} \partial_{s} \phi(s, u, 0)$ which satisfies the $\mathrm{sG}$ equation in the straight region in the absence of driving $\mathcal{I}$ $=0$. Here $X_{0}$ is the initial position, and $v_{0}$ is the initial velocity. For the system of equations (32), satisfied by the collective coordinates, the initial conditions are

$$
S(0)=X_{0}, \dot{S}(0)=v_{0} \quad \text { and } B(0)=\dot{B}(0)=0 .
$$
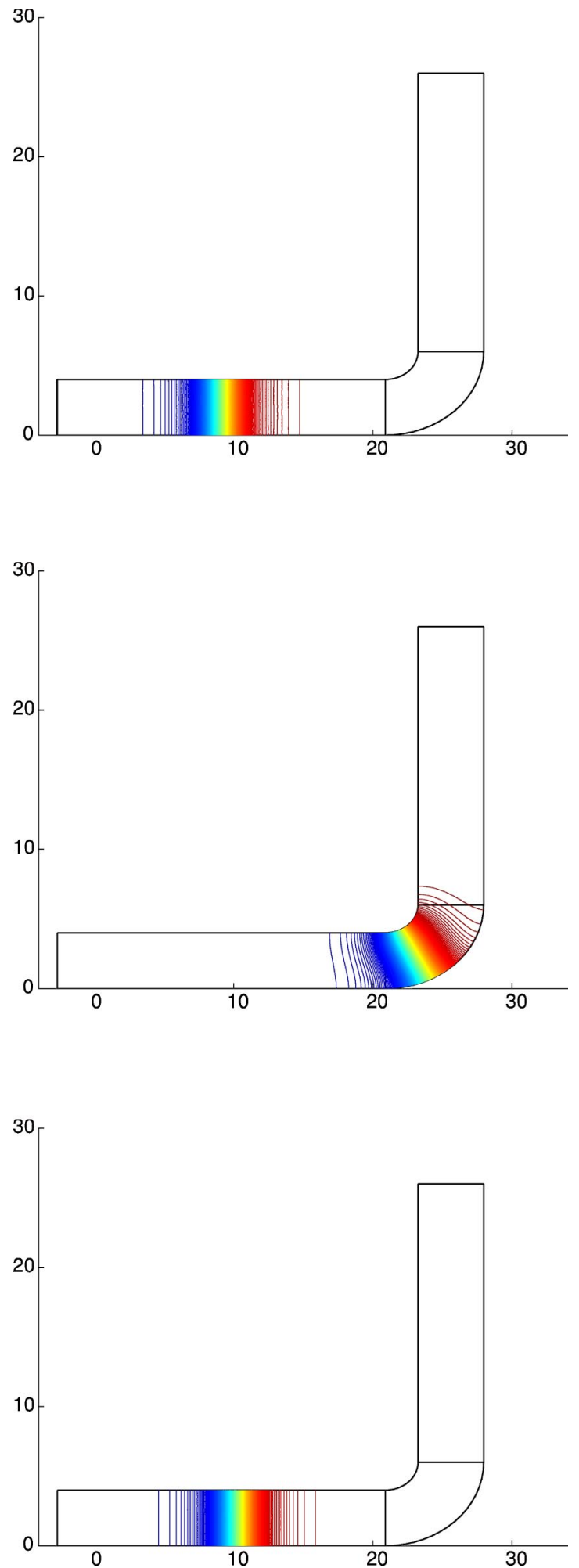

FIG. 6. The wave is reflected for $R=4, w=4$, and $v_{0}=0.17$, while $v_{c r} \approx 0.19$.

Let us consider the kink dynamics in the right-angle waveguide with $w=R=4, s_{2}-s_{1}=2 \pi$. In terms of the collective coordinate approach this choice of parameters corresponds to a rather shallow interhump well (see Fig. 5 dashed line). Two typical examples of the kink evolution for two 

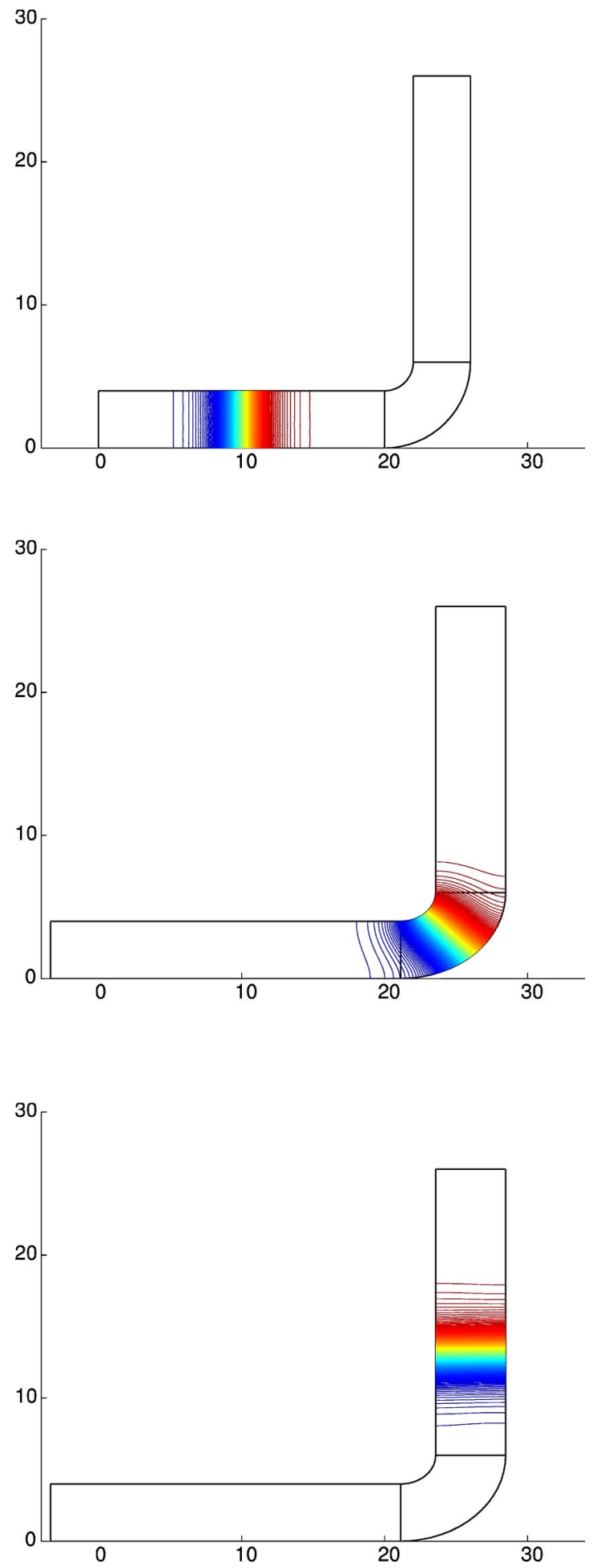

FIG. 7. The wave is transmitted for $R=4, w=4$, and $v_{0}$ $=0.18$, while $v_{c r} \approx 0.19$.

different initial velocities are presented in Figs. 6 and 7. In the first one, corresponding to a small initial velocity, the kink is seen to be reflected from the bending region while in the second, for large enough initial velocity, the kink passes the bent region leaving behind some small radiation.

The results of a more systematic study of the front propagation [i.e., the position of the points for the level $\phi(s, u, t)$

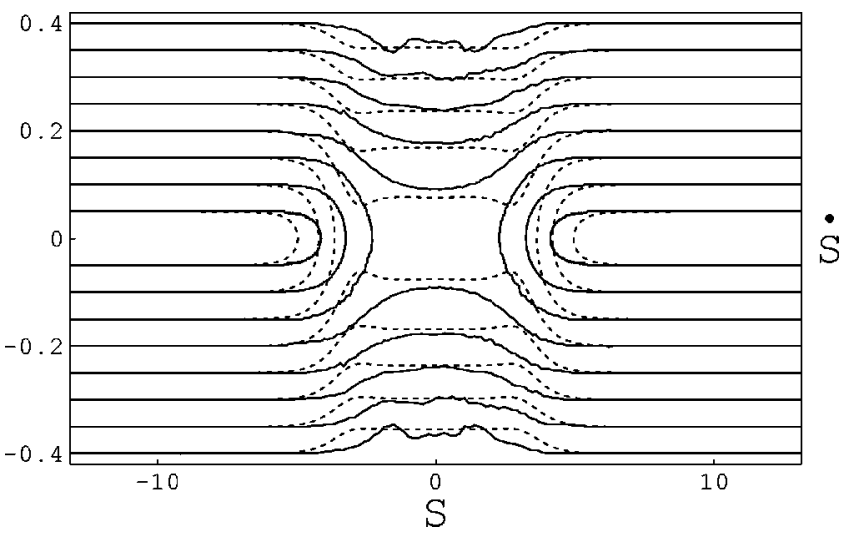

FIG. 8. Phase portrait $(S, \dot{S})$ using $w=4, R=4$ for the simulations (continuous line) and the collective variable equations (37) (dashed line). The critical velocity for transmission is $v_{c r} \approx 0.18$.

$=\pi]$ in the curved stripe are given in the phase portrait [front position $S(t)$, front velocity $\dot{S}$ ] presented in Fig. 8. In the same figure the results of the collective coordinate approach based on Eqs. (32) are also presented. It is observed that the collective coordinate approach gives a qualitatively good agreement with the results of the numerical simulations. As the figure shows, the initial velocity of the soliton determines completely its trajectory. When the initial velocity is lower than some critical value $v_{c r}$ the trajectories do not penetrate into the bending region. On the other hand, for higher initial velocities, $v_{0}>v_{c r}$, the kink passes the curved region and propagates all the way through the junction. In this regard, the motion of sG kinks in two-dimensional curved strips is similar to the propagation of fluxons in Josephson lines with impurities. ${ }^{20}$ It is also worth noting that the approach based on the two variational parameters $S$ and $B$ agrees quantitively with the results of the numerical simulations for the critical velocity $v_{c r}$ while the approach based on a single variational parameter (the kink position) exaggerates the height of the effective potential in the bent region. For example, when $w$ $=R=4$ the value of the critical velocity obtained from simulations is $0.18 \leqslant v_{c r} \leqslant 0.19$, while the variational approach assuming a modulation of the width gives $v_{c r} \approx 0.19$. The simple variational approach based only on the kink position gives $v_{c r} \approx 0.3$.

For a straight driven damped sine-Gordon equation the excitation switches to a spatially uniform state for high-bias current at a critical velocity. ${ }^{22}$ It is an interesting question whether a similar limiting velocity exists in the curved geometry.

\section{B. Case with driving and damping: kink capture}

We consider the motion of fluxons in the presence of damping and driving. The energy-providing mechanism here is modeled by the inhomogeneous von Neumann boundary conditions (6). The equivalence of bending to a potential barrier, demonstrated above, suggests that in a waveguide kinks can be captured nearby the bending by applying an external driving. The $g$ coefficient in the wave equation (7) for the $s$ variable is similar to a surface inductance in the 


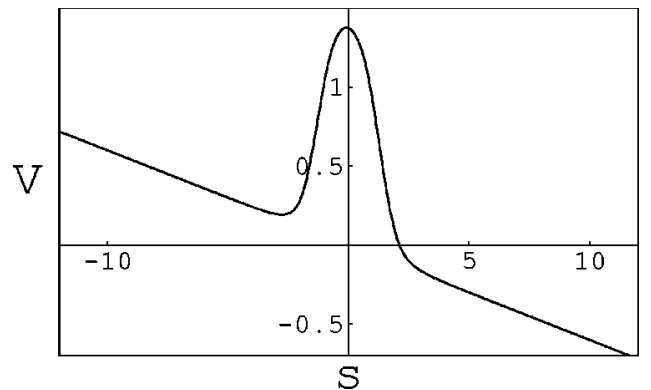

FIG. 9. Effective potential $V(S)$ as a function of the kink positions in the precence of a driving force obtained from Eqs. (27) and (37) in the absence of damping. $\gamma=0.03$ for $w=3, R=2$. Kink trapping is possible as shown by the local minimum of this potential for $S<0$.

Josephson context so that one expects kink trapping at the interface in the presence of a current as in (Ref. 23). Indeed, in the presence of driving and damping the equations of motion for the collective coordinates $S$ and $B$ are (see Appendix B for details)

$$
\begin{gathered}
\ddot{S}+\frac{1}{8} \frac{\partial V}{\partial S}+\alpha \dot{S}+\gamma=0, \\
\ddot{B}+\frac{3}{\pi^{2}} \frac{\partial V}{\partial B}+\alpha \dot{B}=0 .
\end{gathered}
$$

An analysis shows that for $|\gamma|<\gamma_{c r}$ Eqs. (37) possess a stable sink-type fixed point which corresponds to a trapping of fluxon (see Fig. 9). The trap is always located outside the bent region and gets closer to the barrier when the driving force $\gamma$ increases. The critical value of the driving force $\gamma_{c r}$ decreases when the width of the junction decreases (see Figs. $10-11)$.

The two-dimensional projections of the phase portrait onto the $(S, \dot{S})$ plane are shown in Fig. 12. In full agreement with the results of the collective coordinate approach based on Eqs. (37), as the width of the junction increases, the trapping occurs closer to the bent region of the junction.

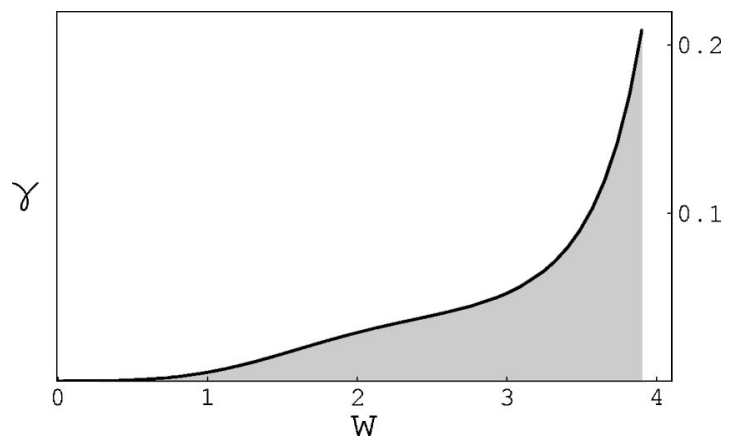

FIG. 10. Phase diagram for $R=2$. The kink-trapping region is shown in gray.

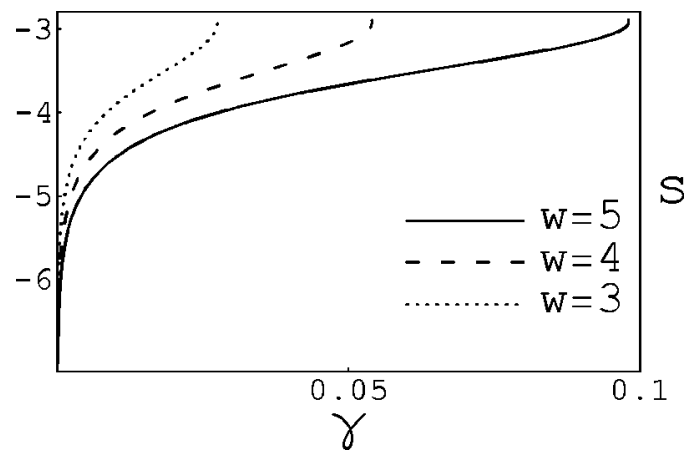

FIG. 11. Position of the kink trap as a function of the current density for three different waveguide widths $w=3,4$, and $5 . R$ $=4$.

\section{CONCLUSIONS}

We consider nonlinear wave propagation in a curved planar waveguide using as a model kink solutions for the sineGordon equation. The waveguide consists of two rectangular regions joined by a bent section of constant curvature. Transverse homogeneous and inhomogeneous Neumann boundary conditions are used. The latter models an energy-providing mechanism for Josephson junctions of overlap type. We develop a collective variable approach based on the kink position and the kink width depending on the transversal coordinate. It allows us to take into account both longitudinal and centrifugal forces, which act on the nonlinear excitation moving in a region with finite curvature and to obtain a qualitatively good agreement with the results of the numerical simulations.

The curved region might manifest itself as a two-hump potential barrier with interbarrier space acting as a potential valley. The height of the barriers and depth of the interbarrier valley depend on the ratio between the width of the waveguide $w$ and its radius of curvature $R$. The appearance of the double barrier structures is due to the two-dimensional character of the kink evolution in curved waveguides. When $w$ $\leqslant R$ the well is shallow and the bending region acts as a potential barrier.

Kink capture may occur when a driving force is applied to

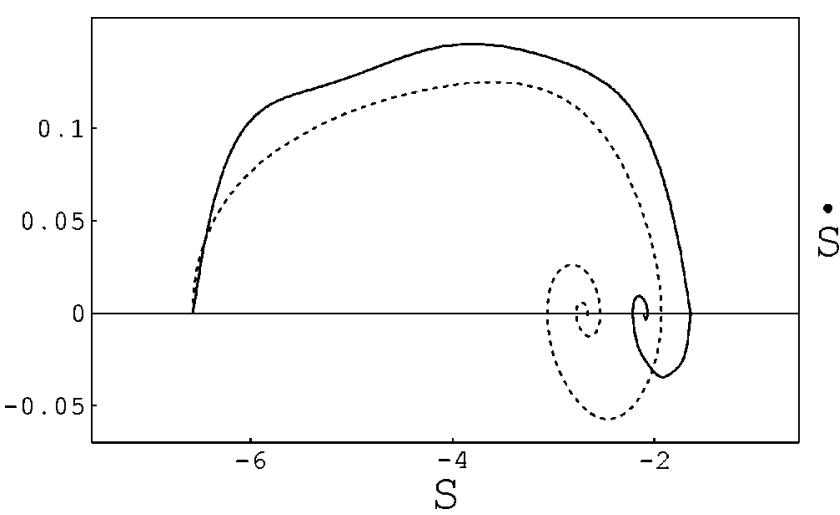

FIG. 12. Numerical (continuous line) and analytical (dashed line) $(S, \dot{S})$ phase portrait for $w=3, R=2, \gamma=0.03$, and $\alpha=0.03$. Trapping occurs at $s<-a=-\pi / 2$. 
the kink. In Josephson junctions this driving force is provided by a magnetic field via the inhomogeneous von Neumann boundary conditions. The kink trapping occurs only in a finite interval of the driving parameter when this force drives the kink against the repulsion caused by the barrier created in the bent section. Beyond this interval the kink is transmitted along the waveguide.

This study shows that by changing the geometry of waveguides one can efficiently control the dynamics of nonlinear excitations. Depending on the width of the waveguide and its curvature the bending regions may act either as potential barriers or as traps for nonlinear excitations. This feature could be applied to electronic devices for storing binary data.

\section{ACKNOWLEDGMENTS}

Yu.B.G. thanks the Department of Informatics and Mathematical Modeling, Technical University of Denmark for the guest professorship and C.G. acknowledges the hospitality of the Technical University of Denmark, helping with all kind of facilities during the investigation period. Financial support was provided by the LOCNET Program (Grant No. HPRNCT-1999-00163), the MIDIT Center, Danish Natural Research Council (Grant No. 21-02-0500) and the project (Grant No. UPV00100.310-E-14806/2002) from the University of the Basque Country. Y.B.G. acknowledges partial support fromDeutsche Zentrum für Luft- und Raumfart e.V., Internatioles Büro des Bundesministeriums für Forschung und Technologie, Bonn, in the frame of a bilateral scientific cooperation between Ukraine and Germany, Project No. UKR02/011.

\section{APPENDIX A}

Inserting Eq. (20) into the Lagrange functions (16)-(19) and carrying out the integration over the arclength $s$, we obtain an effective Lagrangian for the dynamics of the front $\mathcal{S}(u, t)$ and the width $\mathcal{B}(u, t)$ in the form

$$
L\{\phi\}=L\{B, S\}=L_{s}+L_{b}+L_{d r},
$$

where

$$
\begin{aligned}
L_{s}= & 4 \frac{1}{w} \int_{-w / 2}^{w / 2}\left\{\left(\frac{\partial \mathcal{S}}{\partial t}\right)^{2}-\left(\frac{\partial \mathcal{S}}{\partial u}\right)^{2}+\frac{\pi^{2}}{12}\left[\left(\frac{\partial \mathcal{B}}{\partial t}\right)^{2}-\left(\frac{\partial \mathcal{B}}{\partial u}\right)^{2}\right]\right. \\
& \left.-\mathcal{B}^{2}-1\right\} \frac{1}{\mathcal{B}} d u
\end{aligned}
$$

is the effective Lagrange function for the straight junction. The first perturbation term

$$
\begin{aligned}
L_{b}= & -\frac{4}{w} \int_{-w / 2}^{w / 2} \frac{u}{R}\left\{\left[\left(\frac{\partial S}{\partial t}\right)^{2}-\left(\frac{\partial S}{\partial u}\right)^{2}-\mathcal{B}^{2}+\frac{R}{R-u}\right] \mathcal{U}_{0}\right. \\
& \left.+2\left[\frac{\partial S}{\partial t} \frac{\partial B}{\partial t}-\frac{\partial S}{\partial u} \frac{\partial B}{\partial u}\right] \mathcal{U}_{1}+\left[\left(\frac{\partial \mathcal{B}}{\partial t}\right)^{2}-\left(\frac{\partial \mathcal{B}}{\partial u}\right)^{2}\right] \mathcal{U}_{2}\right\} \frac{1}{\mathcal{B}} d u
\end{aligned}
$$

is due to the presence of the bending region (9) in the junction. Here the notation

$$
\mathcal{U}_{n}=\frac{1}{2} \int_{-(a+\mathcal{S}) / \mathcal{B}}^{(a-\mathcal{S}) / \mathcal{B}} x^{n} \operatorname{sech}^{2} x d x, \quad n=0,1,2
$$

is introduced. The second perturbation term also consists of two parts

$$
L_{d r}=L_{d r}^{\prime}+L_{d r}^{\prime \prime}
$$

The functional

$$
L_{d r}^{\prime}=-4 \frac{1}{w} \int_{-w / 2}^{w / 2}\left\{\frac{\pi}{2} \frac{d^{2} \Phi_{0}}{d u^{2}} \mathcal{S}(u, t)-\left(1-\cos \Phi_{0}\right) \mathcal{B}(u, t)\right\} d u
$$

describes the driving effects in the straight junction while

$$
\begin{aligned}
L_{d r}^{\prime \prime}= & \frac{1}{w} \int_{-w / 2}^{w / 2} \frac{1}{R}\left\{\left[\arctan \left(\frac{\sinh (a / \mathcal{B})}{\cosh (\mathcal{S} / \mathcal{B})}\right) \frac{d}{d u}\left(u \frac{d \Phi_{0}}{d u}\right) \mathcal{S}(u, t)\right.\right. \\
& +C(\mathcal{S}, \mathcal{B}) \mathcal{B}(u, t)]+\frac{8 \sinh (a / \mathcal{B}) \sinh (\mathcal{S} / \mathcal{B})}{\cosh (2 a / \mathcal{B})+\cosh (2 \mathcal{S} / \mathcal{B})} u \frac{d^{2} \Phi_{0}}{d u^{2}} \\
& \left.-a \arctan \left(\frac{\cosh (a / \mathcal{B})}{\sinh (\mathcal{S} / \mathcal{B})}\right) \frac{d \Phi_{0}}{d u}\right\} d u
\end{aligned}
$$

with

$$
C(\mathcal{S}, \mathcal{B})=2 \int_{-(a+\mathcal{S}) / \mathcal{B}}^{(a-\mathcal{S}) / \mathcal{B}} x \operatorname{sech} x d x
$$

is due to the combined action of driving force and curvature.

To take explicitly into account the boundary conditions (21) we expand the front $\mathcal{S}(u, t)$ and the width $\mathcal{B}(u, t)$ in Fourier series

$$
\begin{aligned}
\mathcal{S}(u, t)= & \sum_{n=0}^{\infty}\left\{S_{2 n}(t) \cos \left(\frac{2 n \pi u}{w}\right)\right. \\
& \left.+S_{2 n+1}(t) \sin \left(\frac{(n+1) \pi u}{w}\right)\right\}, \\
\mathcal{B}(u, t)= & \sum_{n=0}^{\infty}\left\{B_{2 n}(t) \cos \left(\frac{2 n \pi u}{w}\right)\right. \\
& \left.+B_{2 n+1}(t) \sin \left(\frac{(n+1) \pi u}{w}\right)\right\} .
\end{aligned}
$$

The results of the numerical simulations show that the curvature of the front is small and therefore we need only take into account the first few terms in the expansion (23). In this appendix, to check the accuracy of the approximations used in the main text we extend the set of variational parameters and derive the collective coordinate Lagrangian assuming that

$$
\mathcal{S}(u, t)=S_{0}(t)+S_{1}(t) \sin \left(\frac{\pi u}{w}\right),
$$




$$
\mathcal{B}(u, t)=B_{0}(t)+B_{1}(t) \sin \left(\frac{\pi u}{w}\right) .
$$

First, we consider the case without driving $(\mathcal{I}=0)$ and without damping. Inserting Eqs. (A10) into the Lagrangian (A1)(A3) and carrying out the integrations over the transversal variable, we obtain that the kink dynamics in the case without driving and damping is described by the effective Lagrangian function

$$
\begin{aligned}
L= & \frac{4}{B_{0}}\left\{\dot{S}_{0}^{2}+\frac{1}{2} \dot{S}_{1}^{2}+\frac{\pi^{2}}{12}\left(\dot{B}_{0}^{2}+\frac{1}{2} \dot{B}_{1}^{2}\right)-\frac{\pi^{2}}{2 w^{2}} S_{1}^{2}-B_{0}^{2}-1\right. \\
& -\frac{1}{2 B_{0}^{2}}\left(1+\frac{\pi^{4} B_{0}^{2}}{12 w^{2}}\right) B_{1}^{2}-\frac{2 w}{\pi^{2} R}\left[2 \dot{S}_{0} \dot{S}_{1} U_{0}\right. \\
& \left.+\left(\dot{S}_{0} \dot{B}_{1}+\dot{S}_{1} \dot{B}_{0}\right) U_{1}+2 \dot{B}_{0} \dot{B}_{1} U_{2}\right]-a_{0} U_{0}-a_{1} S_{1} \frac{\partial U_{0}}{\partial S_{0}} \\
& -a_{1} \frac{B_{1}}{B_{0}}\left(B_{0} \frac{\partial U_{0}}{\partial B_{0}}-U_{0}\right)+\frac{2 w}{\pi^{2} R}\left[S_{1} B_{0} \frac{\partial U}{\partial S_{0}}\right. \\
& \left.\left.+B_{1}\left(B_{0} \frac{\partial U_{0}}{\partial B_{0}}+U_{0}\right)\right]\right\},
\end{aligned}
$$

where

$$
U_{n}=\left.\mathcal{U}_{n}\right|_{B_{1}=S_{1}=0}, \quad n=0,1,2 .
$$

We solved numerically the set of Euler-Lagrange equations corresponding to the Lagrangian (A11). The results are presented in Fig. 13 together with the corresponding results obtained in the framework of the simple approach used in the Sec. III. A good agreement of both approaches is observed. Therefore, to gain a qualitative understanding of the kink dynamics it is sufficient to use the simple collective coordinate approach based on two variational parameters $S(t)$ $\equiv S_{0}(t)$ and $B(t) \equiv B_{1}(t)$.

\section{APPENDIX B}

In this appendix we derive a collective coordinate equation of motion for the case with driving and damping. In our derivation we will follow the method given in Ref. 21 for the case of the damped nonlinear Schrödinger equation. The equation of motion (13) can be written in the form

$$
\frac{\partial \mathcal{L}}{\partial \psi}-\frac{\partial}{\partial t} \frac{\partial \mathcal{L}}{\partial \psi_{t}}-\frac{\partial}{\partial s} \frac{\partial \mathcal{L}}{\partial \psi_{s}}-\frac{\partial}{\partial u} \frac{\partial \mathcal{L}}{\partial \psi_{u}}=\alpha g \psi_{t},
$$

where the Lagrange density $L$ is given by Eqs. (17)-(19). Assuming that the solution of the equation may be written as

$$
\psi=4 \arctan \exp \left[\frac{s-S(t)}{1+B(t) \sin (\pi u / w)}\right],
$$

invoking Eq. (16) and integrating by parts we get
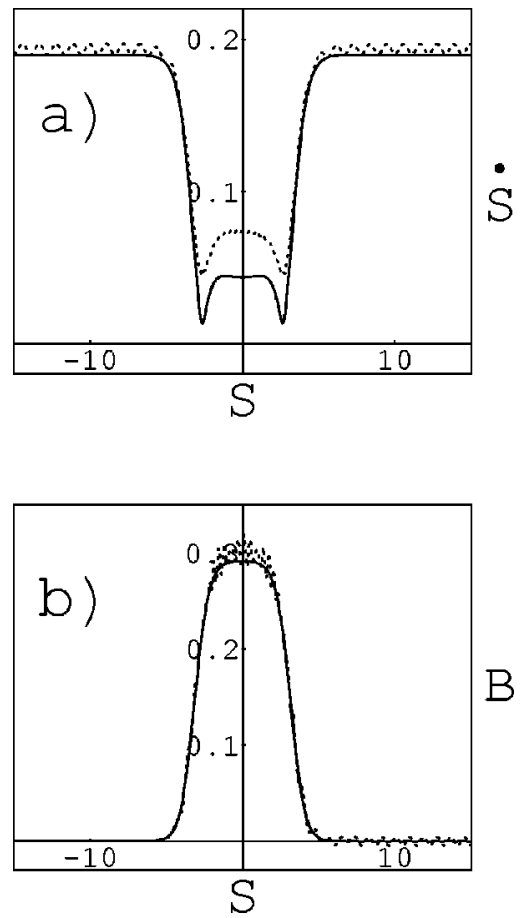

FIG. 13. Comparison of the two-dimensional projections onto $(S, \dot{S})$ plane (upper panel) and onto $(S, B)$ plane (lower panel) for the simple collective coordinate approach, $S(t)=S_{0}(t)$ and $B(t)$ $=B_{1}(t)$ (full curve) and for the extended approach given by Eq. (A10) (dotted curve). $w=4, R=4$.

$$
\begin{aligned}
\frac{\partial L}{\partial S}= & \int_{-\infty}^{\infty} \frac{1}{w} \int_{-w / 2}^{w / 2}\left(\frac{\partial \mathcal{L}}{\partial \psi} \frac{\partial \psi}{\partial S}+\frac{\partial \mathcal{L}}{\partial \psi_{u}} \frac{\partial \psi_{u}}{\partial S}+\frac{\partial \mathcal{L}}{\partial \psi_{s}} \frac{\partial \psi_{s}}{\partial S}\right. \\
& \left.+\frac{\partial \mathcal{L}}{\partial \psi_{t}} \frac{\partial \psi_{t}}{\partial S}\right) d s d u \\
= & \int_{-\infty}^{\infty} \frac{1}{w} \int_{-w / 2}^{w / 2}\left[\left(\frac{\partial \mathcal{L}}{\partial \psi}-\frac{\partial}{\partial u} \frac{\partial \mathcal{L}}{\partial \psi_{u}}-\frac{\partial}{\partial s} \frac{\partial \mathcal{L}}{\partial \psi_{s}}\right) \frac{\partial \psi}{\partial S}\right. \\
& \left.+\frac{\partial \mathcal{L}}{\partial \psi_{t}} \frac{\partial \psi_{t}}{\partial S}\right] d s d u,
\end{aligned}
$$

$$
\begin{aligned}
\frac{d}{d t} \frac{\partial L}{\partial S_{t}} & =\int_{-\infty}^{\infty} \frac{1}{w} \int_{-w / 2}^{w / 2} \frac{\partial \mathcal{L}}{\partial \psi_{t}} \frac{\partial \psi_{t}}{\partial S_{t}} d s d u \\
& =\frac{d}{d t} \int_{-\infty}^{\infty} \frac{1}{w} \int_{-w / 2}^{w / 2} \frac{\partial \mathcal{L}}{\partial \psi_{t}} \frac{\partial \psi}{\partial S} d s d u \\
& =\int_{-\infty}^{\infty} \frac{1}{w} \int_{-w / 2}^{w / 2}\left(\frac{\partial \psi}{\partial S} \frac{d}{d t} \frac{\partial \mathcal{L}}{\partial \psi_{t}}+\frac{\partial \mathcal{L}}{\partial \psi_{t}} \frac{\partial \psi_{t}}{\partial S}\right) d s d u .
\end{aligned}
$$

Subtracting now Eq. (B4) from Eq. (B3) and invoking Eq. (B1), we get

$$
\frac{\partial L}{\partial S}-\frac{d}{d t} \frac{\partial L}{\partial S_{t}}=\alpha \int_{-\infty}^{\infty} \frac{1}{w} \int_{-w / 2}^{w / 2} \frac{\partial \psi}{\partial t} \frac{\partial \psi}{\partial S} g d s d u
$$

In the same way one can obtain the equation for $B$ 


$$
\frac{\partial L}{\partial B}-\frac{d}{d t} \frac{\partial L}{\partial B_{t}}=\alpha \int_{-\infty}^{\infty} \frac{1}{w} \int_{-w / 2}^{w / 2} \frac{\partial \psi}{\partial t} \frac{\partial \psi}{\partial B} g d s d u
$$

Introducing Eq. (B1) into Eqs. (B5)-(B6) and carrying out the integrations with respect to $s$ and $u$ we get

$$
\frac{\partial L}{\partial S}-\frac{d}{d t} \frac{\partial L}{\partial S_{t}}=8 \alpha S_{t},
$$

$$
\frac{\partial L}{\partial B}-\frac{d}{d t} \frac{\partial L}{\partial B_{t}}=\frac{\pi^{2}}{3} \alpha B_{t},
$$

where we neglected the difference of the damping effects in the bent and in the straight regions of the channel due to smallness of the damping coefficient $(\alpha \ll 1)$. The nonlinear terms in the right-hand sides of Eqs. (B5) and (B6) were also neglected.
${ }^{1}$ Photonic Crystals and Light Localization in the 21st Century, Vol. 563 of NATO Advanced Studies Institute, Series C: Mathematical and Physical Sciences, edited by C.M. Soukoulis (Kluwer Academic, 2001).

${ }^{2}$ Nanostructure Physics and Fabrication, edited by M. Reed and W. Kirks (Academic Press, New York, 1989).

${ }^{3}$ T. Shinjo, T. Okuno, R. Hassdorf, K. Shigeto, and T. Ono, Science 289, 930 (2000).

${ }^{4}$ M. Kl ui, C.A.F. Vaz, J. Rothman, J.A.C. Bland, W. Wernsdorfer, G. Faini, and E. Cambril, Phys. Rev. Lett. 90, 097202 (2003).

${ }^{5}$ R.L. Schult, D.G. Ravenhall, and H.W. Wyld, Phys. Rev. B 39, 5476 (1989).

${ }^{6}$ J. Goldstone and R.L. Jaffe, Phys. Rev. B 45, 14100 (1992).

${ }^{7}$ Y.B. Gaididei and O.O. Vakhnenko, J. Phys.: Condens. Matter 6, 32229 (1994).

${ }^{8}$ A. Mekis, S. Fan, and J.D. Joannopoulos, Phys. Rev. B 58, 4809 (1998).

${ }^{9}$ T. Harayama, P. Davis, and K.S. Ikeda, Phys. Rev. Lett. 82, 3803 (1999).

${ }^{10}$ A. Wallraff, A.V. Ustinov, V.V. Kurin, I.A. Shereshevsky, and N.K. Vdovicheva, Phys. Rev. Lett. 84, 151 (2000).

${ }^{11}$ A. Benabdallah, J.G. Caputo, and A.C. Scott, Phys. Rev. B 54,
16139 (1996).

${ }^{12}$ S.F. Mingaleev, Y.S. Kivshar, and R.A. Sammut, Phys. Rev. E 62, 5777 (2000).

${ }^{13}$ Y.B. Gaididei, S.F. Mingaleev, and P.L. Christiansen, Phys. Rev. E 62, R53 (2000).

${ }^{14}$ A.E. Leanhardt, A.P. Chikkatur, D. Kielpinski, Y. Shin, T.L. Gustavson, W. Ketterle, and D.E. Pritchrad, Phys. Rev. Lett. 89, 040401 (2002).

${ }^{15}$ A. Barone and G. Paterno, Physics and Applications of the Josephson Effect (Wiley, New York, 1982).

${ }^{16}$ R. Vaglio, J. Low Temp. Phys. 25, 229 (1976).

${ }^{17}$ P. Exner and P. Seba, J. Math. Phys. 30, 2574 (1989).

${ }^{18}$ J.G. Caputo, N. Flytzanis, Y. Gaididei, and E. Vavalis, Phys. Rev. B 54, 2092 (1996).

${ }^{19}$ www.comsol.com

${ }^{20}$ P.L. Christiansen and O.H. Olsen, Wave Motion 4, 163 (1985).

${ }^{21}$ J.G. Caputo, N. Flytzanis, and M.P. Soerensen, J. Opt. Soc. Am. B 12, 139 (1995).

${ }^{22}$ S. Pagano, M.P. Soerensen, P.L. Christiansen, and R.D. Parmentier, Phys. Rev. B 38, 4677 (1988).

${ }^{23}$ A. Benabdallah, J.G. Caputo, and N. Flytzanis, Physica D 161, 79 (2002). 